OPEN ACCESS

Edited by:

Luigi Iuliano,

Sapienza University of Rome, Italy

Reviewed by:

Steven E. Trasino,

Hunter College, CUNY, USA

Douglas Mashek,

University of Minnesota, USA

*Correspondence:

Amnon Schlege

amnons@u2m2.utah.edu

Specialty section:

This article was submitted to

Lipidology,

a section of the journal

Frontiers in Physiology

Received: 07 February 2017 Accepted: 18 April 2017

Published: 09 May 2017

Citation:

Benitez-Santana T, Hugo SE and Schlegel A (2017) Role of Intestinal LXRa in Regulating Post-prandial Lipid

Excursion and Diet-Induced Hypercholesterolemia and Hepatic

Lipid Accumulation.

Front. Physiol. 8:280.

doi: 10.3389/fphys.2017.00280

\section{Role of Intestinal LXR $\alpha$ in Regulating Post-prandial Lipid Excursion and Diet-Induced Hypercholesterolemia and Hepatic Lipid Accumulation}

\author{
Tibiábin Benitez-Santana ${ }^{1,2}$, Sarah E. Hugo ${ }^{1,2}$ and Amnon Schlegel ${ }^{1,2,3,4 *}$ \\ ${ }^{1}$ University of Utah Molecular Medicine Program, School of Medicine, University of Utah, Salt Lake City, UT, USA, ${ }^{2}$ Division of \\ Endocrinology, Metabolism and Diabetes, Department of Internal Medicine, School of Medicine, University of Utah, Salt Lake \\ City, UT, USA, ${ }^{3}$ Department of Biochemistry, School of Medicine, University of Utah, Salt Lake City, UT, USA, ${ }^{4}$ Department of \\ Nutrition and Integrative Physiology, College of Health, University of Utah, Salt Lake City, UT, USA
}

Post-prandial hyperlipidemia has emerged as a cardiovascular risk factor with limited therapeutic options. The Liver $X$ receptors (Lxrs) are nuclear hormone receptors that regulate cholesterol elimination. Knowledge of their role in regulating the absorption and handling of dietary fats is incomplete. The purpose of this study was to determine the role of intestinal Lxra in post-prandial intestinal lipid transport. Using Lxra knockout ( $n r 1 h^{-/-}$) and intestine-limited Lxro over-expressing [Tg(fabp2a:EGFP-nr1h3)] zebrafish strains, we measured post-prandial lipid excursion with live imaging in larvae and physiological methods in adults. We also conducted a long-term high-cholesterol dietary challenge in adults to examine the chronic effect of modulating $n r 1 h 3$ gene dose on the development of hypercholesterolemia and hepatic lipid accumulation. Over-expression of Lxro in the intestine delays the transport of ingested lipids in larvae, while deletion of $L x r \alpha$ increases the rate of lipid transport. Pre-treating wildtype larvae with the liver-sparing Lxr agonist hyodeoxycholic acid also delayed the rate of intestinal lipid transport in larvae. In adult males, deletion of Lxra accelerates intestinal transport of ingested lipids. Adult females showed higher plasma Lipoprotein lipase (Lpl) activity compared to males, and lower post-gavage blood triacylglycerol (TAG) excursion. Despite the sexually dimorphic effect on acute intestinal lipid handling, Tg(fabp2a:EGFP-nr1h3) adults of both sexes are protected from high cholesterol diet (HCD)-induced hepatic lipid accumulation, while $n r 1 h 3^{-1-}$ mutants are sensitive to the effects of HCD challenge. These data indicate that intestinal $L x$ r activity dampens the pace of intestinal lipid transport cell-autonomously. Selective activation of intestinal Lxra holds therapeutic promise.

Keywords: Liver X Receptor, intestine, postprandial lipemia, triglycerides, cholesterol, chylomicrons, zebrafish

\section{INTRODUCTION}

Atherosclerosis remains the leading cause of death (Lozano et al., 2012). The major driver of atherosclerosis is increased circulating cholesterol-rich lipoprotein particles (Stamler et al., 1986). Statin drugs, which decrease hepatic cholesterol synthesis and thereby promote hepatic cholesterol-rich lipoprotein particle clearance, are potent reducers of the risk of death 
from atherosclerosis (Cholesterol Treatment Trialists' (CTT) Collaboration et al., 2015). Nevertheless, these drugs do not fully ameliorate the risk of ischemic cardiovascular events, ultimately reflecting gaps in understanding of the drivers of atherosclerotic progression.

The persistence of TAG-rich lipoproteins in the post-prandial state has emerged as a major risk factor for atherosclerosis (Jørgensen et al., 2014; The TG and HDL Working Group of the Exome Sequencing Project et al., 2014). Namely, in large prospective cohort studies, risk of atherosclerosis was found to be attributable to the non-fasting circulating TAG levels, which are a proxy measurement of atherogenic lipoprotein particles (chylomicron remnants, intermediary density lipoprotein, IDL; and small, dense low density lipoprotein particles) that should normally not accumulate in the circulation. Indeed, this population-level investigation has pointed to hereditable defects in vascular clearance of TAG-rich lipoproteins, and driven drug development for Mendelian causes of severely elevated TAG levels (Gaudet et al., 2014). For the general population, there are no effective therapies available to blunt post-prandial hypertriglyceridemia (Nordestgaard and Varbo, 2014).

A better understanding of the molecular cues governing intestinal fat absorption, storage and lipoprotein production may lead to new therapies to decrease post-prandial hyperlipidemia (Dash et al., 2015; Giammanco et al., 2015). A detailed generegulatory scheme governing intestinal lipid physiology is still lacking, although much progress has been made in defining the machinery of lipid absorption and lipoprotein production (Iqbal and Hussain, 2009; Abumrad and Davidson, 2012; Dash et al., 2015). The most apparent gaps in our understand of intestinal lipid handling relate to how and why enterocytes store a substantial fraction of absorbed lipids in cytoplasmic lipid droplets, and the cues that control this storage and subsequent release (Robertson et al., 2003; Zhu et al., 2009; Douglass et al., 2012; Dash et al., 2015; Giammanco et al., 2015).

Lxrs are nuclear hormone receptors whose major endogenous ligands are oxysterols, cholesterol catabolites that accumulate in proportion to cholesterol excess (Janowski et al., 1999), and select sterane intermediates of cholesterol synthesis and bile acids (Song et al., 2000; Yang et al., 2006). When activated by ligands, Lxrs drive a multi-organ gene transcriptional program that induces cholesterol elimination (Calkin and Tontonoz, 2012). Genetic activation of Lxr $\alpha$ in the intestine blunts cholesterol absorption (Lo Sasso et al., 2010) and fatty acid absorption (CruzGarcia and Schlegel, 2014); and drives the absorbed lipids into a cytoplasmic lipid droplet pool (Cruz-Garcia and Schlegel, 2014). Pharmacological activation of intestinal Lxrs also blunts fatty acid absorption and transport (Briand et al., 2016). Conversely, global deletion of mouse Lxr $\alpha$ increases the fraction of dietary cholesterol that is absorbed in the presence of a non-sterol Lxr agonist, an effect not seen when $\operatorname{Lxr} \beta$ is deleted ( $\mathrm{Hu}$ et al., 2012). Since oxysterols are excreted in bile (Mutemberezi et al., 2016), these studies argue that the intestine is exposed to multiple Lxr-activating signals that could dampen lipid transport with every meal. Unfortunately, Lxro activation with synthetic nonsterol ligands up-regulates hepatic lipogenesis and Very Low Density Lipoprotein (VLDL) particle secretion (Repa et al.,
2000; Schultz et al., 2000; Grefhorst et al., 2002; Bradley et al., 2007). This property has impeded Lxr-based drug development (Kirchgessner et al., 2016).

Zebrafish models of dyslipidemia have emerged in recent years as a powerful system for studying intestinal lipid transport, vascular lipoprotein metabolism, and the early steps of atherosclerosis (Fang et al., 2014; Schlegel, 2016). Most notably, zebrafish have similar abundance and distribution of circulating lipoproteins as humans (Stoletov et al., 2009; Liu et al., 2015), unlike numerous preclinical models (Yin et al., 2012). Additionally, examination of intestinal lipid handling has been studied extensively in zebrafish with results concordant with findings seen in Mendelian Diseases (Schlegel and Stainier, 2006; Avraham-Davidi et al., 2012; Levic et al., 2015).

It is important to stress that zebrafish carry a singly Lxr $\alpha$ ortholog, whose encoding gene is syntenic to human LXR $\alpha$ (Reschly et al., 2008; Cruz-Garcia et al., 2009; Fonseca et al., 2017). The Lxr $\beta$ locus was lost in the fish phylum (Fonseca et al., 2017). Furthermore, zebrafish Lxr $\alpha$ binds and is activated by endogenous and synthetic ligands in a manner similar to human LXR $\alpha$ (Archer et al., 2008; Reschly et al., 2008). Finally, human intestines express LXR $\alpha$ only (Uhlén et al., 2015). In this study we examine the effect of modulating zebrafish intestinal Lxr $\alpha$ activation on intestinal lipid transport and on the accumulation of vascular and hepatic lipids using new live imaging and physiological methods on our previously described Lxra deletion and intestinal over-expression lines. We show intestinal Lxr $\alpha$ activation delays transport of ingested lipids, decreasing the postprandial plasma TAG excursion. This effect protects animals from HCD-induced hepatic lipid accumulation. The methods we have optimized will be of broad use to others interested in studying aspects of enterocyte handling of lipids.

\section{METHODS}

\section{Animals}

The Institutional Animal Care and Use Committee of the University of Utah approved all studies. Animals were euthanized by tricaine overdose or immersion in ice. The Centralized Zebrafish Animal Resource (CZAR) at the University of Utah maintains the wildtype (WT) WIK strain zebrafish used in this study. The $n r 1 h 3^{z 101 a}$, and $\operatorname{Tg}(f a b p 2 a: E G F P-n r 1 h 3)^{z 103}$ lines were described previously (Cruz-Garcia and Schlegel, 2014). The $n r 1 h 3^{z 101 a}$ mutant strain was targeted with Transcriptional Activator-like Effector Nucleases to create an in-frame stop mutation within the DNA binding domain (i.e., it is a null mutation). The $\operatorname{Tg}(f a b p 2 a: E G F P-n r 1 h 3)^{z 103}$ line drives Lxr $\alpha$ expression in enterocytes, and animals carrying a single-copy of the transgene (heterozygous) were used in all experiments.

\section{Larval Gavage and Whole-Mount Epifluorescence Microscopy}

Four nanoliters of a 5:1 mixture $(v: v)$ of triolein and cholesteryl oleate containing 1:1000 $(v: v)$ dilution of cholesteryl BODIPY ${ }^{\circledR}$ 542/563 undecanoate ( $\mathrm{CE}_{11}$-BODIPY) was gavaged into the proximal intestines of zebrafish larvae exactly as described (Cocchiaro and Rawls, 2013). After gavage, and 
between live microscopic scoring sessions, animals were maintained at $28^{\circ} \mathrm{C}$, with a 14 -h light:10-h dark cycle. Red fluorescent signal in the vasculature was monitored with a Leica M 205 FA stereomicroscope fitted with a camera and computer in a blinded fashion. Six larvae from each genotype were studied simultaneous, and the results are presented as the mean \pm standard error of the mean $(n=18$ larvae in total per each analysis). For experiments involving hyodeoxycholic acid (HDCA, Sigma), the compound was dissolved in dimethylsulfoxide, and $4 \mu \mathrm{L}$ of vehicle or of a $5 \mu \mathrm{M}$ solution was gavaged $24 \mathrm{~h}$ prior to gavage of the lipid mixture.

\section{Tyloxapol Injection, Oral Lipid Gavage, Lipoprotein Lipase Activity}

Three months post-fertilization (mpf) adult zebrafish were injected intraperitoneally with $2.5 \mathrm{mg} / \mathrm{g}$ tyloxapol (Millar et al., 2005). Animals were then subjected to an oral gavage with $0.1 \mathrm{~mL}$ of a $5: 1(v: v)$ mixture of olive oil and cholesteryl ester. In pilot experiments, plasma TAG peaked $8 \mathrm{~h}$ after oral gavage. Thus, 5 and $8 \mathrm{~h}$ after oral gavage, animals underwent terminal phlebotomy and plasma TAG was measured. Plasma Lpl activity was measured using a commercial kit (Cayman Chemicals, Michigan, USA) exactly as described on plasma collected at the 8-h time point to confirm suppression of Lpl activity throughout the experimental window (Liu et al., 2015). Lpl activity was measured in a 15-min ex vivo assay, and the slope of each activity curve was taken as the rate constant for the Lpl activity present.

\section{High Cholesterol Feeding}

The high cholesterol $(4 \% w / w)$ diet was prepared as previously described (Stoletov et al., 2009). Commercial flakes (TetraMin Tropical Flakes, Blacksburg, VA) were soaked in a cholesteroldiethyl ether solution and the flakes were left to dry overnight. For all adult studies, 5 female and 5 male 3 mpf zebrafish were distributed in $3 \mathrm{~L}$ tanks. The animals were fed twice daily with automatic feeders for 7 months. Never-mated females were housed separately to avoid confounding issues of post-spawning vitellogenesis marked by transient hepatic steatosis (Sheridan, 1988; Babin and Vernier, 1989).

\section{Blood and Tissue Lipid Composition Analysis}

Blood was collected by cardiac puncture with heparinized glass capillaries attached to a microinjector (Microinjection Systems, Harvard System). Blood was diluted in $0.2 \mathrm{~mL}$ tubes with $20 \mu \mathrm{L}$ of PBS-EDTA. After centrifugation at maximum speed for $5 \mathrm{~min}$, plasma was collected. Tissues were homogenized in lysis buffer $(20 \mathrm{mM}$ Tris- $\mathrm{HCl}, 150 \mathrm{mM} \mathrm{NaCl}, 1 \mathrm{mM}$ EDTA, $1 \mathrm{mM}$ EGTA, 1\% Triton X-100) by sonication. Protein concentration was determined with the BCA protein assay reagent (Thermo Scientific). The TAG and total cholesterol levels in the blood were analyzed with colorimetric assay kits (Spinreact, Mexico).

Total lipids were Folch-extracted from liver (Iverson et al., 2001). Unesterified cholesterol, cholesteryl esters, TAG, and free fatty acids were resoled using thin layer chromatography exactly as we described previously (Schlegel and Stainier, 2006; Hugo et al., 2012; Karanth et al., 2013). The abundance of each lipid class was quantified using a standard charring and copper-based densitometric assay (Bitman and Wood, 1982; Ruiz and Ochoa, 1997), with normalization of lipid abundance to protein content (Cruz-Garcia and Schlegel, 2014).

\section{RT-PCR}

RNA was extracted from liver and analyzed for acaca, fasn, $h m g c r a$, srebfl, and srebf2 abundance exactly as we described previously, using the $r p p 0$ transcript for normalization (Karanth et al., 2013; Cruz-Garcia and Schlegel, 2014). Intestinal abca1a and $a b c a 1 b$ abundance was quantified from intestines using $5^{\prime}$-CCACATCGAGGACTACTCCG and 5'-TGTCTCTTTGGC CTTCTCGT; and 5'-TCTCCCAGACCACACTAGACC and 5'TTTGGTCCTTCGCAAAGTTT, respectively.

\section{Statistical Analysis}

Statistical analyses were performed using DataGraph 4.1 (Visual Data Tools) and SPSS 19.0 (IBM) software. Data are presented as means \pm standard error of the mean. The normality of the variable distribution was verified using Levene's test; and the data did not require transformation. Unless explicitly stated otherwise, differences with the WT group were evaluated using Student's $t$-test or 1-way ANOVA, with indicated parametric tests. A significance of $P<0.05$ was applied to all statistical tests performed.

\section{RESULTS}

\section{Lxr $\alpha$ Gene Dose Regulates the Rate of Transport of Gavaged Lipids in Zebrafish Larvae}

Intestinal Lxr $\alpha$ over-expression induces a gene expression program that diverts absorbed lipids into a cytoplasmic lipid droplet pool (Cruz-Garcia and Schlegel, 2014); however, wholemount Oil Red O (ORO) histological staining proved insensitive in revealing differences in vascular lipid accumulation between WT and $n r 1 h 3^{-/-}$larvae (Cruz-Garcia and Schlegel, 2014). Thus, we performed oral gavage to deliver a defined bolus of neutral lipids and the fluorescent lipid $\mathrm{CE}_{11}$ - BODIPY, whose fatty acyl chain can be incorporated into neutral and phospholipids in vivo (Carten et al., 2011), into the proximal intestine of zebrafish larvae (Figure 1A). Immediately after gavage, fluorescent signal was seen only in the lumen of the intestine, and starting at $5 \mathrm{~h}$ post-gavage, fluorescent signal could be seen in the vasculature (Figure 1B). After gavage, $n r 1 h 3^{-/-}$mutant larvae showed more rapid vascular lipid accumulation compared to WT and $\operatorname{Tg}($ fabp2a:EGFP-nr1h3) larvae (Figure 1C). All WT and $n r 1 h 3^{-/-}$mutant larvae showed vascular fluorescent lipid accumulation by $10.5 \mathrm{~h}$ after oral gavage, while approximately one-third of $\operatorname{Tg}(f a b p 2 a$ :EGFPnrlh3) transgenic larvae had no vascular lipid accumulation. These results, obtained by repeated imaging of live larvae, confirm and extend our previous findings with fixed larvae, and revealed a difference between WT and $n r 1 h 3^{-/-}$mutants that was not apparent when examining animals fed a lipid rich meal 


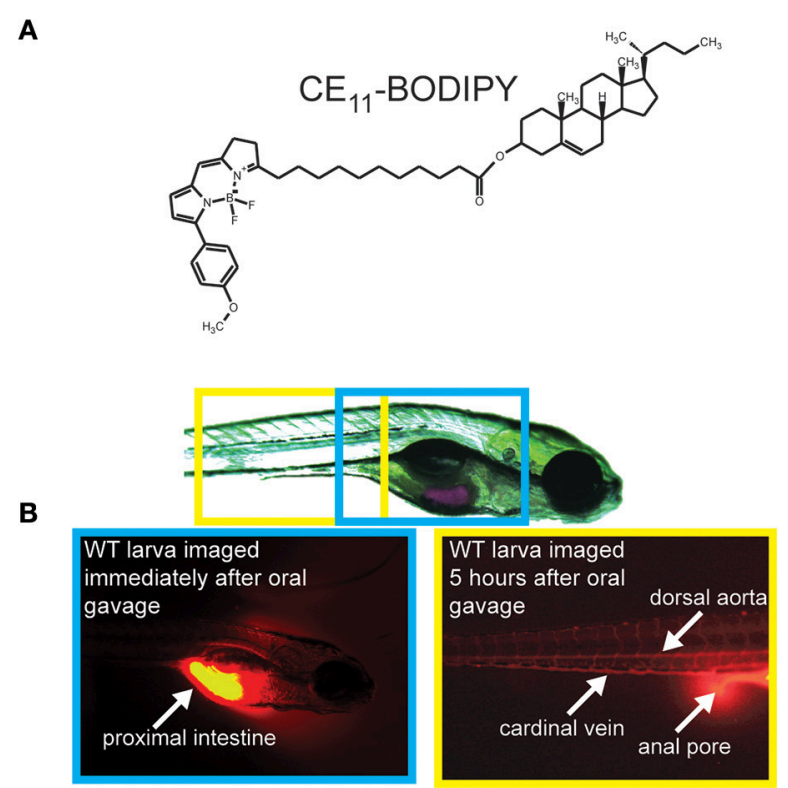

C

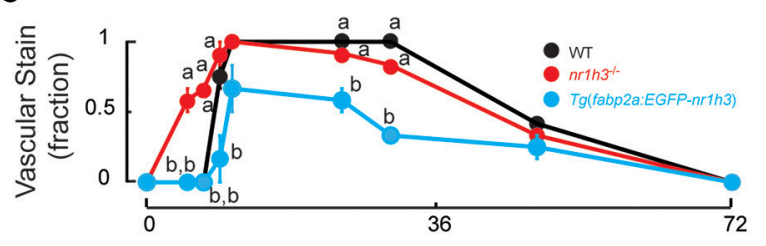

Time Post-Gavage (hours)

D

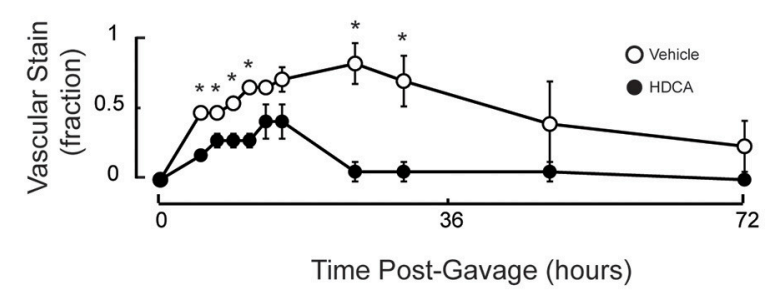

FIGURE 1 | Genetic activation of intestinal Lxr $\alpha$ regulates the pace of transport of ingested lipids in larvae. (A) Structure of $\mathrm{CE}_{11}$-BODIPY. (B) A 7 days post-fertilization (dpf) larva following oral gavage of a mixture of triolein, cholesteryl oleate and $\mathrm{CE}_{11}$-BODIPY (upper). Immediately after oral gavage, the fluorescent signal is very strong in the anterior intestine (lower left). Within 5 $\mathrm{h}$ of gavage, strong vascular staining is apparent, and excess (not absorbed) label can be seen passing through the anal pore (lower right). (C) Six dpf WT, $n r 13^{-/-}$, and Tg(fabp2a:EGFP-nr1h3) transgenic larvae underwent gavage with the lipid mixture, and were scored for the appearance of lipids in the vasculature in a blinded fashion. Values not sharing a common superscript letter are significantly different at $P<0.05$ at each time point in 2-sided student $t$-tests vs. WT; $n=18$ for each genotype. (D) Five dpf WT larvae underwent gavage with vehicle or HDCA. Twenty-four hours later larvae were gavaged with the lipid mixture, and were scored for the appearance of lipids in the vasculature in a blinded fashion. ${ }^{*} P<0.05$ at each time point in 2 -sided student $t$-tests vs. WT; $n=18$ for each genotype.

and then fixed and stained with ORO (Cruz-Garcia and Schlegel, 2014).

We previously found using a less sensitive ORO-based staining method that $n r 1 h 3^{-/-} ; \operatorname{Tg}(f a b p 2 a: E G F P-n r 1 h 3)$ larvae showed delayed appearance of lipids in the vasculature following a fatty meal (Cruz-Garcia and Schlegel, 2014). This finding suggested that enterocyte over-expressed Lxr $\alpha$ acts cell autonomously to regulate the pace of chylomicron appearance. This genetic finding and the results in Figure 1C, nevertheless, raise the possibility that life-long changes in gene expression in other tissues might contribute to the pace of dietary lipid transport. To address this issue, we subjected WT larvae to oral gavage with the liver-sparing (i.e., does not induce de novo lipogenesis) Lxro agonist HDCA (Singhal et al., 1984; CohenSolal et al., 1995; Song et al., 2000; Sehayek et al., 2001; Shih et al., 2013; De Marino et al., 2017). Twenty-four hours later, we performed a second oral gavage of the fluorescently labeled lipid mixture. Figure 1D shows that compared to pre-gavage with vehicle, HDCA pre-gavage delayed the appearance of fluorescent lipid in the vasculature and blunted the peak fraction of animals with vascular lipid staining. Thus, acute intestinal Lxro activation is sufficient to blunt transport of absorbed lipids.

\section{Adult Zebrafish Have Sexually Dimorphic Plasma Lpl Activity}

To test whether the differences among the three cohorts of larval persist into adulthood, we measured post-gavage TAG in animals injected intraperitoneally with the Lpl inhibitor tyloxapol (Millar et al., 2005) at a concentration that inhibited serum Lpl activity for the duration of the experimental window. This standard approach avoids the potential contribution of differential lipolysis to the measured TAG. When injected with PBS, we found that females showed higher plasma Lpl activity in this ex vivo assay compared to males, with $\operatorname{Tg}($ fabp2a:EGFP-nr1h3) transgenic females showing the highest activity (Figure 2A). Tyloxapol successfully inhibited Lpl activity in both sexes and among all genotypes; however, the decreases in Lpl activity following tyloxapol injection were only significantly lower in females (Figure 2A).

\section{Adult Male Zebrafish Have Greater Post-Gavage Lipid Excursion, Which Is Modulated by Lxr $\alpha$ Gene Dose}

After confirming complete inhibition of Lpl over the course of the experimental window, we measured post-gavage plasma TAG excursions. Male $n r 1 h 3^{-/-}$animals showed the greatest increase in post-gavage serum TAG concentrations, at both time points examined (Figure 2B). Both $T g(f a b p 2 a$ :EGFP-nr1h3) males and females showed decreased post-gavage serum TAG at both 5 and $8 \mathrm{~h}$ after gavage, although the difference was only significant in males compared to $n r 1 h 3^{-/-}$animals. These results, while revealing a previously unreported sexual dimorphism in zebrafish plasma Lpl activity, recapitulate the larval phenotypes we observed.

\section{Adult Zebrafish Lacking Lxr $\alpha$ Show Severe Hypercholesterolemia after HCD Challenge}

Next, we conducted a long HCD-feeding experiment to examine the effect of modulating intestinal Lxr $\alpha$ gene 

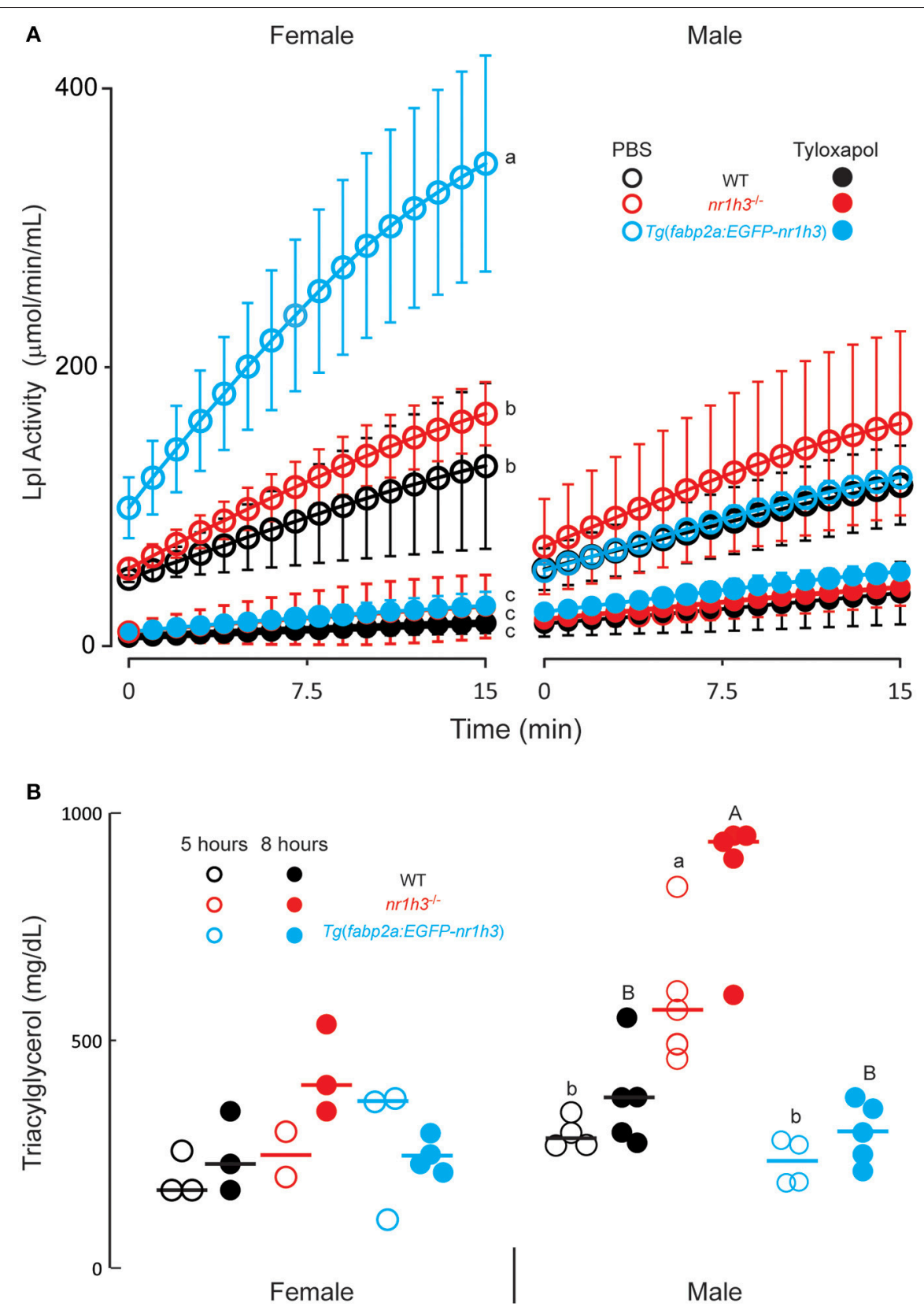

FIGURE 2 | Genetic activation of intestinal Lxr $\alpha$ regulates the pace of transport of ingested lipids in adults. (A) Lipoprotein lipase (Lpl) activity $8 \mathrm{~h}$ after intraperitoneal injection of PBS or Tyloxapol ( $n=6$ for each line). (B) Plasma TAG concentration at the indicated times following oral gavage of lipids. Values not sharing a common superscript letter are significantly different at $P<0.05$ in 1-way ANOVA.

dose on the development of dyslipidemia and hepatic lipid accumulation. The $n r 1 h 3^{-/-}$animals developed severe hypercholesterolemia, with a median value over $2,000 \mathrm{mg} / \mathrm{dL}$ at the conclusion of the HCD feeding period (Figure $\mathbf{3 A}$ ). WT and $\operatorname{Tg}($ fabp2a:EGFP-nr1h3) animals were protected from hypercholesterolemia to a similar degree. The plasma HDL cholesterol (Figure 3B) and TAG (Figure 3C) were not significantly different among the three genotypes on either diet.
Adult Zebrafish Lacking Lxr $\alpha$ Show Severe Hepatic Lipid Accumulation, While Animals Over-Expressing Lxr $\alpha$ in the Intestine Are Protected from Hepatic Lipid Accumulation The livers of $n r 1 h 3^{-/-}$animals had significant increases in free and esterified cholesterol concentrations under HCD feeding, while $\operatorname{Tg}(f a b p 2 a: E G F P-n r 1 h 3)$ livers had significantly lower values (Figures 4A,B). Hepatic free fatty acids were significantly 


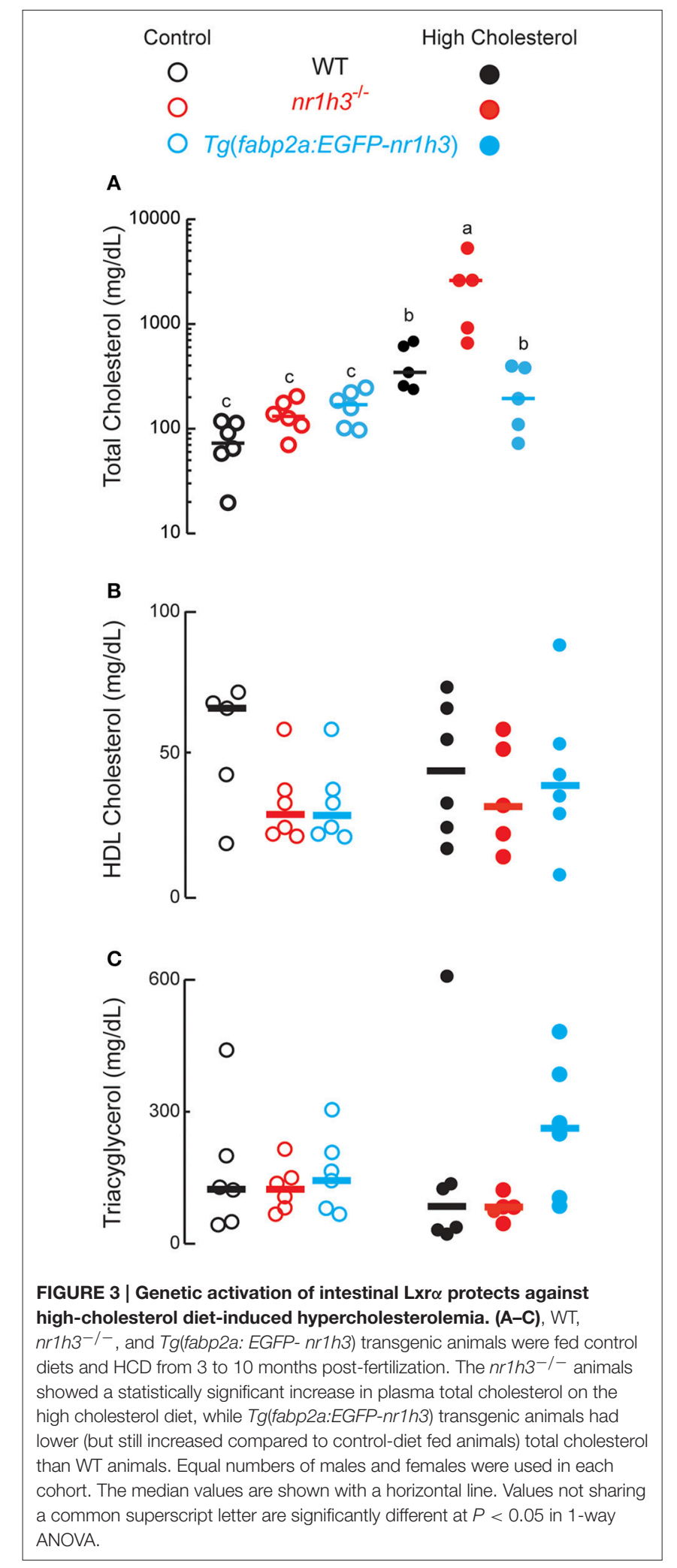

lower in $\operatorname{Tg}($ fabp2a:EGFP-nr1h3) animals; however, diet did not affect this difference (Figure 4C). Liver TAG were significantly lower in $\mathrm{Tg}(f a b p 2 a: E G F P-n r 1 h 3)$ livers compared to $n r 1 h 3^{-/-}$

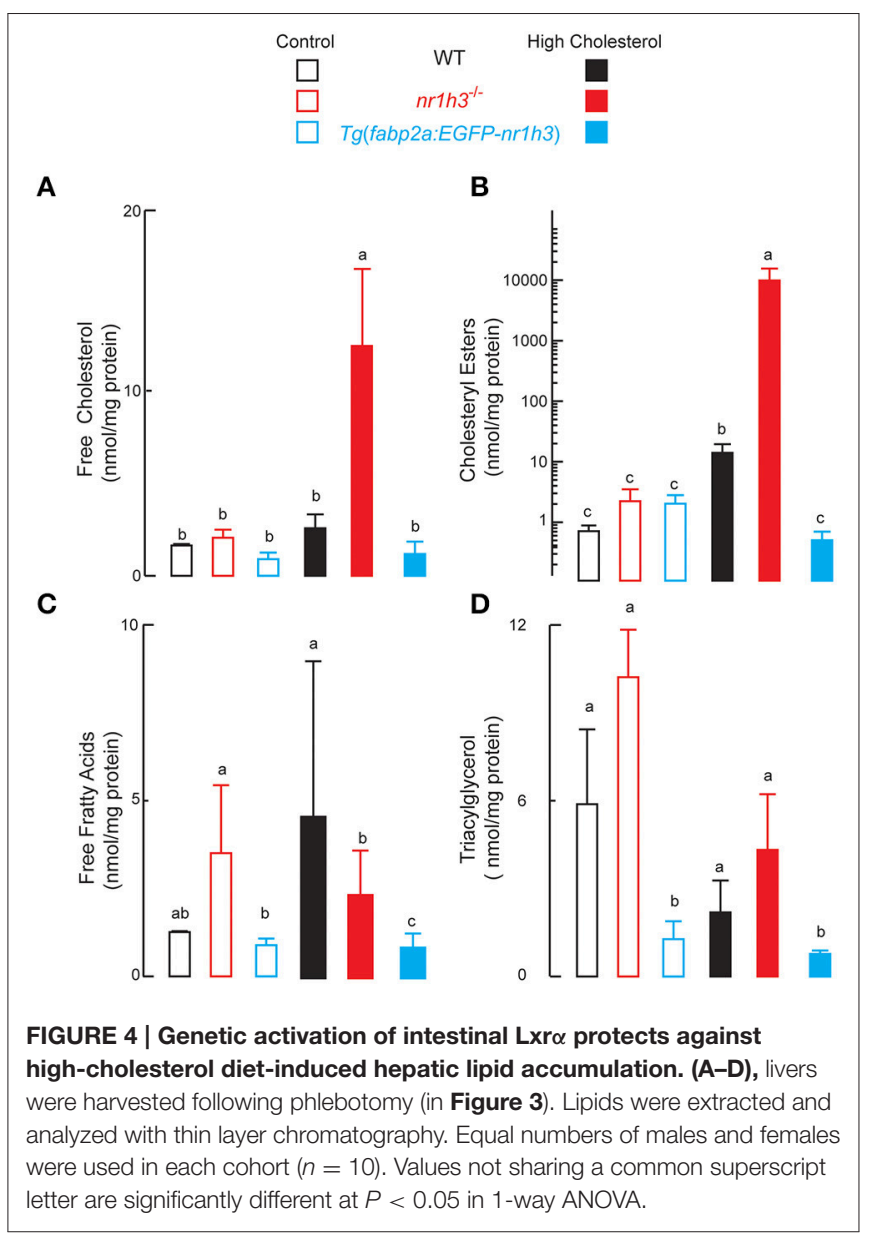

animals, and there were significant differences between dietary cohorts, with all three genotypes showing lower liver TAG under HCD (Figure 4D). These fasting blood and liver lipid parameters were not sexually dimorphic, and are shown in aggregate for both sexes.

Next, we measured the abundance of abcala and $a b c a 1 b$ transcripts in intestines to assess the effect of intestinal Lxro overexpression on the expression of the basolateral sterol exporter that loads cholesterol onto HDL (Repa et al., 2000; Murthy et al., 2002). As expected, intestines $n r 1 h 3^{-/-}$animals had lower expression of both $a b c 1 a$ and $a b c 1 b$. $T g(f a b p 2 a: E G F P-n r 1 h 3)$ intestines from females showed increased $a b c 1 b$ abundance (Figure 5A).

Finally, because hepatic Lxro is critical for cholesterol elimination, and it drives de novo lipogenesis (Zhang et al., 2012), we were curious to examine the effects of intestinal Lxr $\alpha$ overexpression on hepatic gene expression of srebf 1 , srebf 2 encoding the master transcriptional regulators of de novo lipogenesis and cholesterol biosynthesis, respectively, and representative target genes, acaca, fasn, and hmgcra. Critically, srebf1 is a key direct target of Lxro (Schultz et al., 2000; Rong et al., 2017). Figure 5B shows that srebf1 and its two targets acaca and fas $n$ were induced in $n r 1 h 3^{-/-}$livers; while srebf 2 and its target gene hmgcra were induced in $\operatorname{Tg}($ fabp2a:EGFP-nr1h3) livers. 
A

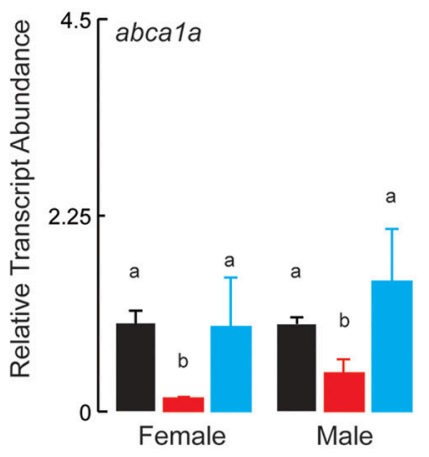

B

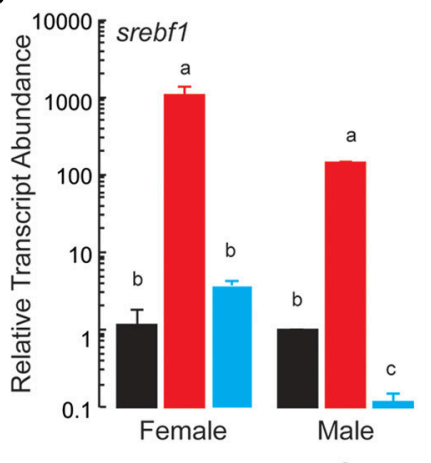

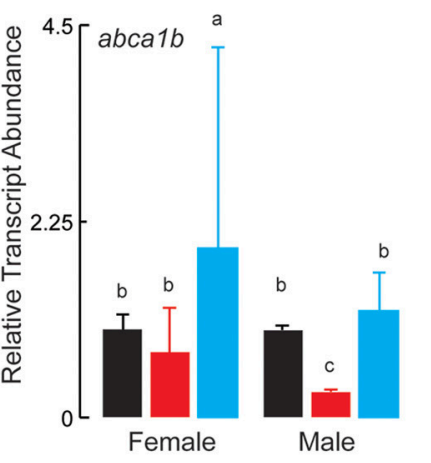

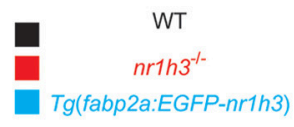

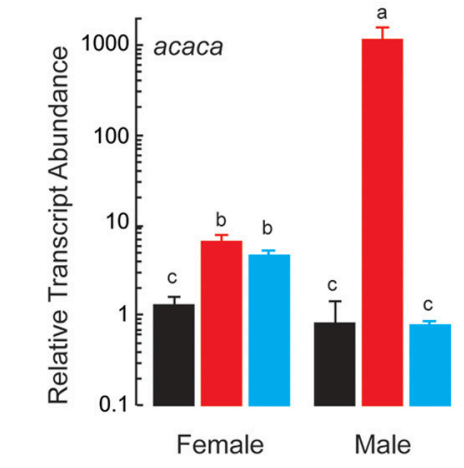
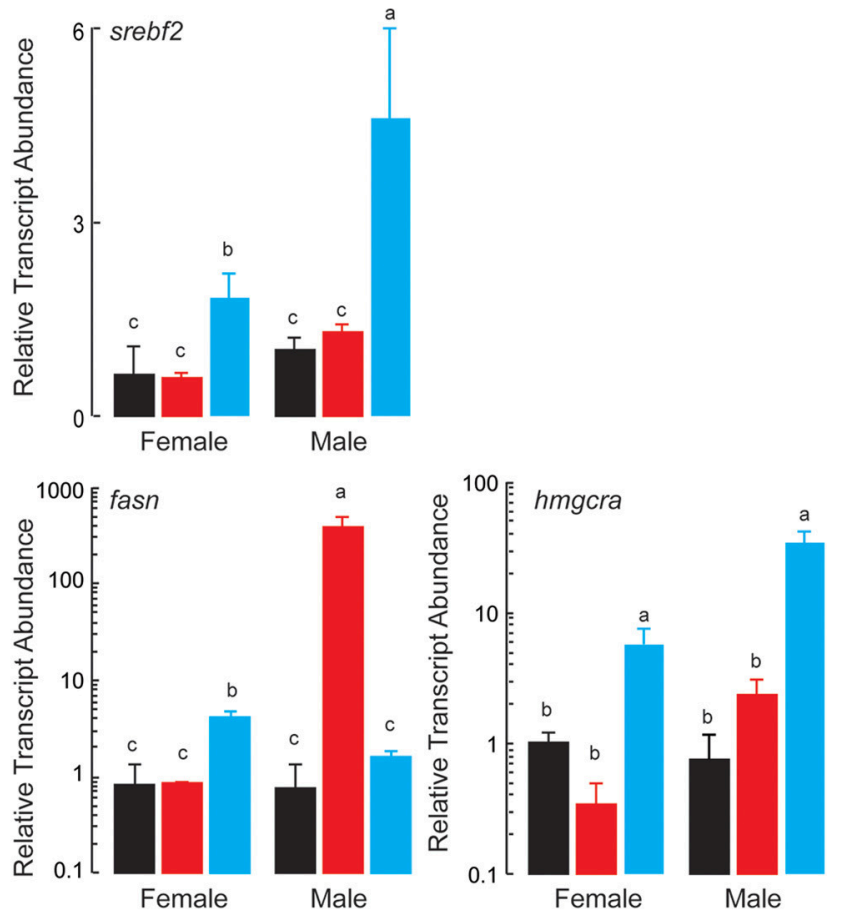

FIGURE 5 | Expression of Transcripts Encoding Components of Intestinal HDL Synthesis and Hepatic De Novo Lipogenesis. (A) Livers were dissected from adult animals ( $n=3$ per sex) and srebf1, srebf2, fasn, and acaca transcripts were measured. Values not sharing a common superscript letter are significantly different at $P<0.05$. (B) Intestines were dissected from adult animals $(n=3$ per sex) fed the high cholesterol diets. RNA was extracted and abca1a and abca1b transcripts were quantified. Values not sharing a common superscript letter are significantly different at $P<0.05$ in 1 -way ANOVA.

\section{DISCUSSION}

The intestine can serve as a reservoir for absorbed dietary lipids in metazoans ranging from insects to fish, rodents, and humans (Robertson et al., 2003; Sieber and Thummel, 2009; Zhu et al., 2009; Douglass et al., 2012). The molecular signals controlling this storage function are beginning to emerge. We previously demonstrated that zebrafish Lxro cell-autonomously regulates intestinal handling of absorbed lipids, with over-expression of Lxr $\alpha$ (i.e., increased activation relying on endogenous ligands only) diverting absorbed lipids to an enterocyte cytoplasmic storage pool by inducing expression of the long-chain acylCoA ligase gene acsl3, which encodes a lipid droplet-targeted enzyme that funnels CoA thioesters of fatty acids to neutral and phospholipids (Cruz-Garcia and Schlegel, 2014).

Here we developed novel methods for live imaging of larvae and biochemical assessment in adults to monitor lipid transport in zebrafish. This work was motivated by our observation that whole mount ORO staining was not sufficiently sensitive to discern differences between WT and $n r 1 h 3^{-/-}$animals. In larvae we found using a more sensitive live imaging approach that intestinal over-expression of Lxr $\alpha$ delays and deletion of Lxr $\alpha$ increases the rate of transport of orally gavaged fluorescent lipid tracers. This relatively facile approach in larvae should be of use in future genetic and pharmacological studies to explore not only the consequences of modulating Lxr target gene dose, but in 
studying the effects of drugs on intestinal lipid handling. Since there is modest larval manipulation and serial measurement required (i.e., gavage, and repeated live imaging, with periods of free swimming at the euthermal temperature and normal light cycle conditions), a preclinical validation platform could emerge from the use of our methods. A moderate-scale chemical screen could also be based on this workflow with zebrafish larvae (Clifton et al., 2010).

In adults, we observed a sexually dimorphic role for Lxr $\alpha$ in regulating the rate of transport of absorbed lipids. Specifically, we found that genetic activation of intestinal Lxr $\alpha$ delayed the appearance of lipids in the circulation acutely in males. Differences in intestinal sterol transporters do not appear to account for these differences. Rather, there appears to be sexually dimorphic, intestinal Lxr $\alpha$-directed differences in plasma Lpl activity. The molecular cues regulating the higher Lpl activity and relatively blunted post-prandial lipemic excursion in female zebrafish will require further study; however, it is important to note that women also have higher Lpl activity than men (Mittendorfer et al., 2003). This relative protection from post-prandial hyperlipidemia is reminiscent of the human epidemiological observations of relative cardiac protection in premenopausal women (Lozano et al., 2012). It is also reminiscent of the long-standing observation that $17-\beta$-estradiol protects ovariectomized female mice from atherosclerotic progression in Apoe $e^{-/-}$mice (Bourassa et al., 1996). Restoration of this physiological estrogen with subcutaneous pellets reduces total plasma cholesterol, VLDL/IDL cholesterol, and TAGs (Bourassa et al., 1996). Remarkably, 17- $\beta$-estradiol protects $\mathrm{Ldlr}^{-/-}$mice from atherosclerosis; however, the effect does not appear to be related to changes in circulating lipoproteins, as measured in the non-fasting state (Marsh et al., 1999). Thus, there appear to be context-dependent atheroprotective roles for $17-\beta$-estradiol in mice. In future studies, we will determine whether the sexually dimorphic post-prandial lipid excursion in zebrafish impacts atherogenesis. In both sexes, intestinal overexpression of Lxr $\alpha$ in the intestine blunted the development of hepatic cholesterol accumulation during a long HCD challenge, a feeding paradigm in which Lxro deletion caused severe hypercholesterolemia in both sexes. Since our experimental approach fully suppressed Lpl activity in females and males, there are probably additional sexual modifiers of postprandial lipid excursion that merit additional study.

More generally, our results in a zebrafish model of Lxr $\alpha$ deletion and cell type-limited over-expression are important because postprandial dyslipidemia is not amenable to most currently available lipid-lowering therapies (Nordestgaard and

\section{REFERENCES}

Abumrad, N. A., and Davidson, N. O. (2012). Role of the gut in lipid homeostasis. Physiol. Rev. 92, 1061-1085. doi: 10.1152/physrev.00019.2011

Archer, A., Lauter, G., Hauptmann, G., Mode, A., and Gustafsson, J.-Å. (2008). Transcriptional activity and developmental expression of liver $\mathrm{X}$ receptor (lxr) in zebrafish. Dev. Dyn. 237, 1090-1098. doi: 10.1002/dvdy.21476
Varbo, 2014). Our results suggest that selective activation of Lxr $\alpha$ in the intestine might serve to treat this condition. The atherosclerosis that emerges from repeated, prolonged bouts of exposure to TAG-rich lipoprotein particles in the post-prandial state might be ameliorated by intestine-limited Lxro activation. Similar to HDCA, cholane and cholestane Lxr agonists might be useful for this purpose: while such ligands induce transcription of the master transcription factor driving de novo lipogenesis Srebf1, they simultaneously block Srebf1 proteolytic maturation by stabilizing the precursor in the endoplasmic reticulum, thereby avoiding the induction of hypertriglyceridemia and hepatic steatosis (Kaneko et al., 2003; Quinet et al., 2004; Peng et al., 2008, 2011; Kratzer et al., 2009). Non-sterol ligands lack the biophysical properties to arrest Srebf1 maturation, and their administration is marked by hepatic steatosis and hypertriglyceridemia (Schultz et al., 2000; Grefhorst et al., 2002; Bradley et al., 2007; Kirchgessner et al., 2016). Full dissection of the transcriptional program controlled by intestinal Lxro, as well as dissection the reasons for the sexually dimorphic traits seen in our study, will provide a thorough mechanistic basis for developing new therapies that leverage the capacity of the intestine to store (and safely oxidize) absorbed fatty acids, while promoting net cholesterol elimination.

Finally, we note that the severe hypercholesterolemia seen in the $n r 1 h 3^{-/-}$mutant animals fed the HCD provides a unique system for studying atherosclerosis: this degree of non-HDL cholesterol increase was achieved without additional genetic manipulations, reflecting conservation of aspects of lipoprotein metabolism other established models lack (Yin et al., 2012), such as the retention of an ortholog of the human Cholesteryl Ester Transfer Protein gene (Schlegel, 2016). We anticipate the liveimagine and adult physiology methods described here will allow the zebrafish model to be useful for preclinical testing of a range of lipid-lowering therapies.

\section{AUTHOR CONTRIBUTIONS}

TB and AS designed the study, analyzed the data and wrote the paper. TB and SH performed experiments. All authors discussed the results and commented on the manuscript.

\section{FUNDING}

This work was supported by a Grant In Aid from the Western States Affiliate of the American Heart Association to AS. (15GRNT24670009). Grunspan, M., Malkinson, G., et al. (2012). ApoB-containing lipoproteins regulate angiogenesis by modulating expression of VEGF receptor 1. Nat. Med. 18, 967-973. doi: 10.1038/nm. 2759

Babin, P. J., and Vernier, J. M. (1989). Plasma lipoproteins in fish. J. Lipid Res. 30, 467-489. 
Bitman, J., and Wood, D. L. (1982). An improved copper reagent for quantitative densitometric thin-layer chromatography of lipids. J. Liquid Chromatogr. 5, 1155-1162. doi: 10.1080/01483918208067575

Bourassa, P. A., Milos, P. M., Gaynor, B. J., Breslow, J. L., and Aiello, R. J. (1996). Estrogen reduces atherosclerotic lesion development in apolipoprotein E-deficient mice. Proc. Natl. Acad. Sci. U.S.A. 93, 10022-10027. doi: 10.1073/pnas.93.19.10022

Bradley, M. N., Hong, C., Chen, M., Joseph, S. B., Wilpitz, D. C., Wang, X., et al. (2007). Ligand activation of LXR $\beta$ reverses atherosclerosis and cellular cholesterol overload in mice lacking $\operatorname{LXR} \alpha$ and apoE. J. Clin. Invest. 117, 2337-2346. doi: 10.1172/JCI31909

Briand, O., Touche, V., Colin, S., Brufau, G., Davalos, A., Schonewille, M., et al. (2016). Liver X receptor regulates triglyceride absorption through intestinal down-regulation of Scavenger Receptor Class B, Type 1. Gastroenterology 150, 650-658. doi: 10.1053/j.gastro.2015.11.015

Calkin, A. C., and Tontonoz, P. (2012). Transcriptional integration of metabolism by the nuclear sterol-activated receptors LXR and FXR. Nat. Rev. Mol. Cell Biol. 13, 213-224. doi: 10.1038/nrm3312

Carten, J. D., Bradford, M. K., and Farber, S. A. (2011). Visualizing digestive organ morphology and function using differential fatty acid metabolism in live zebrafish. Dev. Biol. 360, 276-285. doi: 10.1016/j.ydbio.2011. 09.010

Clifton, J. D., Lucumi, E., Myers, M. C., Napper, A., Hama, K., Farber, S. A., et al. (2010). Identification of novel inhibitors of dietary lipid absorption using zebrafish. PLoS ONE 5:e12386. doi: 10.1371/journal.pone.0012386

Cocchiaro, J. L., and Rawls, J. F. (2013). Microgavage of zebrafish larvae. J. Vis. Exp. 20:e4434. doi: 10.3791/4434

Cohen-Solal, C., Parquet, M., Férézou, J., Sérougne, C., and Lutton, C. (1995). Effects of hyodeoxycholic acid and $\alpha$-hyocholic acid, two $6 \alpha$-hydroxylated bile acids, on cholesterol and bile acid metabolism in the hamster. Biochim. Biophys. Acta 1257, 189-197. doi: 10.1016/0005-2760(95)00073-L

Cholesterol Treatment Trialists' (CTT) Collaboration, Fulcher, J., O'Connell, R., Voysey, M., Emberson, J., Blackwell, L., et al. (2015). Efficacy and safety of LDL-lowering therapy among men and women: meta-analysis of individual data from 174-000 participants in 27 randomised trials. Lancet 385, 1397-1405. doi: 10.1016/S0140-6736(14)61368-4

Cruz-Garcia, L., Minghetti, M., Navarro, I., and Tocher, D. R. (2009). Molecular cloning, tissue expression and regulation of liver X receptor (LXR) transcription factors of Atlantic salmon (Salmo salar) and rainbow trout (Oncorhynchus mykiss). Comp. Biochem. Physiol. B Biochem. Mol. Biol. 153, 81-88. doi: 10.1016/j.cbpb.2009.02.001

Cruz-Garcia, L., and Schlegel, A. (2014). Lxr-driven enterocyte lipid droplet formation delays transport of ingested lipids. J. Lipid Res. 55, 1944-1958. doi: 10.1194/jlr.M052845

Dash, S., Xiao, C., Morgantini, C., and Lewis, G. F. (2015). New insights into the regulation of chylomicron production. Annu. Rev. Nutr. 35, 265-294. doi: 10.1146/annurev-nutr-071714-034338

De Marino, S., Carino, A., Masullo, D., Finamore, C., Marchianò, S., Cipriani, S., et al. (2017). Hyodeoxycholic acid derivatives as liver $\mathrm{X}$ receptor $\alpha$ and G-protein-coupled bile acid receptor agonists. Sci. Rep. 7:43290. doi: $10.1038 /$ srep 43290

Douglass, J., Malik, N., Chon, S. H., Wells, K., Zhou, Y. X., Choi, A., et al. (2012). Intestinal mucosal triacylglycerol accumulation secondary to decreased lipid secretion in obese and high fat fed nice. Front. Physiol. 3:25. doi: 10.3389/fphys.2012.00025

Fang, L., Liu, C., and Miller, Y. I. (2014). Zebrafish models of dyslipidemia: relevance to atherosclerosis and angiogenesis. Transl. Res. 163, 99-108. doi: 10.1016/j.trsl.2013.09.004

Fonseca, E., Ruivo, R., Lopes-Marques, M., Zhang, H., Santos, M. M., Venkatesh, B., et al. (2017). LXRalpha and LXRbeta nuclear receptors evolved in the common ancestor of gnathostomes. Genome Biol. Evol. 9, 222-230. doi: 10.1093/gbe/evw305

Gaudet, D., Brisson, D., Tremblay, K., Alexander, V. J., Singleton, W., Hughes, S. G., et al. (2014). Targeting APOC3 in the familial chylomicronemia syndrome. N. Engl. J. Med. 371, 2200-2206. doi: 10.1056/NEJMoa1400284

Giammanco, A., Cefalù, A. B., Noto, D., and Averna, M. R. (2015). The pathophysiology of intestinal lipoprotein production. Front. Physiol. 6:61. doi: 10.3389/fphys.2015.00061
Grefhorst, A., Elzinga, B. M., Voshol, P. J., Plösch, T., Kok, T., Bloks, V. W., et al. (2002). Stimulation of lipogenesis by pharmacological activation of the Liver X Receptor leads to production of large, triglyceride-rich Very Low Density Lipoprotein particles. J. Biol. Chem. 277, 34182-34190. doi: 10.1074/jbc.M204887200

Hu, X., Steffensen, K. R., Jiang, Z. Y., Parini, P., Gustafsson, J. Å., Gåfvels, M., et al. (2012). LXR $\beta$ activation increases intestinal cholesterol absorption, leading to an atherogenic lipoprotein profile. J. Int. Med. 272, 452-464. doi: $10.1111 / j .1365-2796.2012 .02529 . x$

Hugo, S. E., Cruz-Garcia, L., Karanth, S., Anderson, R. M., Stainier, D. Y., and Schlegel, A. (2012). A monocarboxylate transporter required for hepatocyte secretion of ketone bodies during fasting. Genes Dev. 26, 282-293. doi: 10.1101/gad.180968.111

Iqbal, J., and Hussain, M. M. (2009). Intestinal lipid absorption. Am. J. Physiol. 296, E1183-E1194. doi: 10.1152/ajpendo.90899.2008

Iverson, S. J., Lang, S. L., and Cooper, M. H. (2001). Comparison of the Bligh and Dyer and Folch methods for total lipid determination in a broad range of marine tissue. Lipids 36, 1283-1287. doi: 10.1007/s11745-001-0843-0

Janowski, B. A., Grogan, M. J., Jones, S. A., Wisely, G. B., Kliewer, S. A., Corey, E. J., et al. (1999). Structural requirements of ligands for the oxysterol liver X receptors LXR $\alpha$ and LXR $\beta$. Proc. Natl. Acad. Sci. U.S.A. 96, 266-271. doi: 10.1073/pnas.96.1.266

Jørgensen, A. B., Frikke-Schmidt, R., Nordestgaard, B. G., and Tybjærg-Hansen, A. (2014). Loss-of-function mutations in APOC 3 and risk of ischemic vascular disease. N. Engl. J. Med. 371, 32-41. doi: 10.1056/NEJMoa1308027

Kaneko, E., Matsuda, M., Yamada, Y., Tachibana, Y., Shimomura, I., and Makishima, M. (2003). Induction of intestinal ATP-binding cassette transporters by a phytosterol-derived liver $\mathrm{X}$ receptor agonist. J. Biol. Chem. 278, 36091-36098. doi: 10.1074/jbc.M304153200

Karanth, S., Tran, V. M., Balagurunathan, K., and Schlegel, A. (2013). Polyunsaturated fatty acyl-Coenzyme As are inhibitors of cholesterol biosynthesis. Dis. Model. Mech. 6, 1365-1377. doi: 10.1242/dmm.013425

Kirchgessner, T. G., Sleph, P., Ostrowski, J., Lupisella, J., Ryan, C. S., Liu, X., et al. (2016). Beneficial and adverse effects of an LXR agonist on human lipid and lipoprotein metabolism and circulating neutrophils. Cell Metab. 24, 223-233. doi: 10.1016/j.cmet.2016.07.016

Kratzer, A., Buchebner, M., Pfeifer, T., Becker, T. M., Uray, G., Miyazaki, M., et al. (2009). Synthetic LXR agonist attenuates plaque formation in apoE $E^{-/-}$ mice without inducing liver steatosis and hypertriglyceridemia. J. Lipid Res. 50, 312-326. doi: 10.1194/jlr.M800376-JLR200

Levic, D. S., Minkel, J. R., Wang, W. D., Rybski, W. M., Melville, D. B., and Knapik, E. W. (2015). Animal model of Sarlb deficiency presents lipid absorption deficits similar to Anderson disease. J. Mol. Med. 93, 165-176. doi: 10.1007/s00109-014-1247-x

Liu, C., Gates, K. P., Fang, L., Amar, M. J., Schneider, D. A., Geng, H., et al. (2015). Apoc2 loss-of-function zebrafish mutant as a genetic model of hyperlipidemia. Dis. Mod. Mech. 8, 989-998. doi: 10.1242/dmm.019836

Lo Sasso, G., Murzilli, S., Salvatore, L., D'errico, I., Petruzzelli, M., Conca, P., et al. (2010). Intestinal specific LXR activation stimulates reverse cholesterol transport and protects from atherosclerosis. Cell Metab. 12, 187-193. doi: 10.1016/j.cmet.2010.07.002

Lozano, R., Naghavi, M., Foreman, K., Lim, S., Shibuya, K., Aboyans, V., et al. (2012). Global and regional mortality from 235 causes of death for 20 age groups in 1990 and 2010: a systematic analysis for the Global Burden of Disease Study 2010. Lancet 380, 2095-2128. doi: 10.1016/S0140-6736(12) 61728-0

Marsh, M. M., Walker, V. R., Curtiss, L. K., and Banka, C. L. (1999). Protection against atherosclerosis by estrogen is independent of plasma cholesterol levels in LDL receptor-deficient mice. J. Lipid Res. 40, 893-900.

Millar, J. S., Cromley, D. A., McCoy, M. G., Rader, D. J., and Billheimer, J. T. (2005). Determining hepatic triglyceride production in mice: comparison of poloxamer 407 with Triton WR-1339. J. Lipid Res. 46, 2023-2028. doi: 10.1194/jlr.D500019-JLR200

Mittendorfer, B., Patterson, B. W., and Klein, S. (2003). Effect of sex and obesity on basal VLDL-triacylglycerol kinetics. Am. J. Clin. Nutr. 77, 573-579.

Murthy, S., Born, E., Mathur, S. N., and Field, F. J. (2002). LXR/RXR activation enhances basolateral efflux of cholesterol in CaCo-2 cells. J. Lipid Res. 43, 1054-1064. doi: 10.1194/jlr.M100358-JLR200 
Mutemberezi, V., Guillemot-Legris, O., and Muccioli, G. G. (2016). Oxysterols: from cholesterol metabolites to key mediators. Prog. Lipid Res. 64, 152-169. doi: 10.1016/j.plipres.2016.09.002

Nordestgaard, B. G., and Varbo, A. (2014). Triglycerides and cardiovascular disease. Lancet 384, 626-635. doi: 10.1016/S0140-6736(14)61177-6

Peng, D., Hiipakka, R. A., Dai, Q., Guo, J., Reardon, C. A., Getz, G. S., et al. (2008). Antiatherosclerotic effects of a novel synthetic tissue-selective steroidal liver $\mathrm{X}$ receptor agonist in low-density lipoprotein receptor-deficient mice. J. Pharmacol. Exp. Ther. 327, 332-342. doi: 10.1124/jpet.108.142687

Peng, D., Hiipakka, R. A., Xie, J.-T., Dai, Q., Kokontis, J. M., Reardon, C. A., et al. (2011). A novel potent synthetic steroidal liver X receptor agonist lowers plasma cholesterol and triglycerides and reduces atherosclerosis in $\mathrm{LDLR}^{-/-}$mice. $\mathrm{Br}$. J. Pharmacol. 162, 1792-1804. doi: 10.1111/j.1476-5381.2011.01202.x

Quinet, E. M., Savio, D. A., Halpern, A. R., Chen, L., Miller, C. P., and Nambi, P. (2004). Gene-selective modulation by a synthetic oxysterol ligand of the liver X receptor. J. Lipid Res. 45, 1929-1942. doi: 10.1194/jlr.M400257JLR200

Repa, J. J., Turley, S. D., Lobaccaro, J. A., Medina, J., Li, L., Lustig, K., et al. (2000). Regulation of absorption and ABC1-mediated efflux of cholesterol by RXR heterodimers. Science 289, 1524-1529. doi: 10.1126/science.289.548 4.1524

Reschly, E. J., Ai, N., Welsh, W. J., Ekins, S., Hagey, L. R., and Krasowski, M. D. (2008). Ligand specificity and evolution of liver X receptors. J. Steroid Biochem. Mol. Biol. 110, 83-94. doi: 10.1016/j.jsbmb.2008.02.007

Robertson, M. D., Parkes, M., Warren, B. F., Ferguson, D. J. P., Jackson, K. G., Jewell, D. P., et al. (2003). Mobilisation of enterocyte fat stores by oral glucose in humans. Gut 52, 834-839. doi: 10.1136/gut.52.6.834

Rong, S., Cortés, V. A., Rashid, S., Anderson, N. N., Mcdonald, J. G., Liang, G., et al. (2017). Expression of SREBP-1c requires SREBP-2-mediated generation of a sterol ligand for LXR in livers of mice. eLife 6:e25015. doi: 10.7554/eLife. 25015

Ruiz, J. I., and Ochoa, B. (1997). Quantification in the subnanomolar range of phospholipids and neutral lipids by monodimensional thin-layer chromatography and image analysis. J. Lipid Res. 38, 1482-1489.

Schlegel, A. (2016). Zebrafish modles for dyslipidemia and atherosclerosis research. Front. Endocrinol. 7:159. doi: 10.3389/fendo.2016.00159

Schlegel, A., and Stainier, D. Y. (2006). Microsomal triglyceride transfer protein is required for yolk lipid utilization and absorption of dietary lipids in zebrafish larvae. Biochemistry 45, 15179-15187. doi: 10.1021/bi0619268

Schultz, J. R., Tu, H., Luk, A., Repa, J. J., Medina, J. C., Li, L., et al. (2000). Role of LXRs in control of lipogenesis. Genes Dev. 14, 2831-2838. doi: 10.1101/gad. 850400

Sehayek, E., Ono, J. G., Duncan, E. M., Batta, A. K., Salen, G., Shefer, S., et al. (2001). Hyodeoxycholic acid efficiently suppresses atherosclerosis formation and plasma cholesterol levels in mice. J. Lipid Res. 42, 1250-1256.

Sheridan, M. A. (1988). Lipid dynamics in fish: aspects of absorption, transportation, deposition and mobilization. Comp. Biochem. Physiol. B 90, 679-690. doi: 10.1016/0305-0491(88)90322-7

Shih, D. M., Shaposhnik, Z., Meng, Y., Rosales, M., Wang, X., Wu, J., et al. (2013). Hyodeoxycholic acid improves HDL function and inhibits atherosclerotic lesion formation in LDLR-knockout mice. FASEB J. 27, 3805-3817. doi: 10.1096/fj.12-223008
Sieber, M. H., and Thummel, C. S. (2009). The DHR96 nuclear receptor controls triacylglycerol homeostasis in Drosophila. Cell Metab. 10, 481-490. doi: 10.1016/j.cmet.2009.10.010

Singhal, A. K., Cohen, B. I., Finver-Sadowsky, J., Mcsherry, C. K., and Mosbach, E. H. (1984). Role of hydrophilic bile acids and of sterols on cholelithiasis in the hamster. J. Lipid Res. 25, 564-570.

Song, C., Hiipakka, R. A., and Liao, S. (2000). Selective activation of liver X receptor alpha by $6 \alpha$-hydroxy bile acids and analogs. Steroids $65,423-427$. doi: 10.1016/S0039-128X(00)00127-6

Stamler, J., Wentworth, D., and Neaton, J. D. (1986). Is relationship between serum cholesterol and risk of premature death from coronary heart disease continuous and graded?: findings in 356222 primary screenees of the multiple risk factor intervention trial (mrfit). JAMA 256, 2823-2828. doi: 10.1001/jama.1986.03380200061022

Stoletov, K., Fang, L., Choi, S. H., Hartvigsen, K., Hansen, L. F., Hall, C., et al. (2009). Vascular lipid accumulation, lipoprotein oxidation, and macrophage lipid uptake in hypercholesterolemic zebrafish. Circ. Res. 104, 952-960. doi: 10.1161/CIRCRESAHA.108.189803

The TG and HDL Working Group of the Exome Sequencing Project, National Heart, Lung, Blood Institute, Crosby, J., Peloso, G. M., Auer, P. L., Crosslin, D. R., Stitziel, N. O., et al. (2014). Loss-of-function mutations in APOC3, triglycerides, and coronary disease. N. Engl. J. Med. 371, 22-31. doi: 10.1056/NEJMoa1307095

Uhlén, M., Fagerberg, L., Hallström, B. M., Lindskog, C., Oksvold, P., Mardinoglu, A., et al. (2015). Proteomics. Tissue-based map of the human proteome. Science 347:1260419. doi: 10.1126/science.1260419

Yang, C., Mcdonald, J. G., Patel, A., Zhang, Y., Umetani, M., Xu, F., et al. (2006). Sterol intermediates from cholesterol biosynthetic pathway as liver X receptor ligands. J. Biol. Chem. 281, 27816-27826. doi: 10.1074/jbc.M603781200

Yin, W., Carballo-Jane, E., McLaren, D. G., Mendoza, V. H., Gagen, K., Geoghagen, N. S., et al. (2012). Plasma lipid profiling across species for the identification of optimal animal models of human dyslipidemia. J. Lipid Res. 53, 51-65. doi: 10.1194/jlr.M019927

Zhang, Y., Breevoort, S. R., Angdisen, J., Fu, M., Schmidt, D. R., Holmstrom, S. R., et al. (2012). Liver $\operatorname{LXR} \alpha$ expression is crucial for whole body cholesterol homeostasis and reverse cholesterol transport in mice. J. Clin. Invest. 122, 1688-1699. doi: 10.1172/JCI59817

Zhu, J., Lee, B., Buhman, K. K., and Cheng, J. X. (2009). A dynamic, cytoplasmic triacylglycerol pool in enterocytes revealed by ex vivo and in vivo coherent anti-Stokes Raman scattering imaging. J. Lipid Res. 50, 1080-1089. doi: 10.1194/jlr.M800555-JLR200

Conflict of Interest Statement: The authors declare that the research was conducted in the absence of any commercial or financial relationships that could be construed as a potential conflict of interest.

Copyright (C) 2017 Benitez-Santana, Hugo and Schlegel. This is an open-access article distributed under the terms of the Creative Commons Attribution License (CC BY). The use, distribution or reproduction in other forums is permitted, provided the original author(s) or licensor are credited and that the original publication in this journal is cited, in accordance with accepted academic practice. No use, distribution or reproduction is permitted which does not comply with these terms. 